\title{
Localization of mRNA for vascular endothelial growth factor (VEGF), angiopoietins and their receptors during the peri-implantation period and early pregnancy in marmosets (Callithrix jacchus)
}

\author{
A. J. Rowe ${ }^{1}$, C. Wulff ${ }^{2}$ and H. M. Fraser ${ }^{1 *}$ \\ ${ }^{1}$ Medical Research Council Human Reproductive Sciences Unit, Centre for Reproductive Biology, \\ University of Edinburgh, Chancellor's Building, 49 Little France Crescent, Edinburgh EH16 4SB, UK; and \\ ${ }^{2}$ Department of Obstetrics and Gynaecology, University of UIm, Prittwitzstrasse 43, \\ 89075 Ulm, Germany
}

\begin{abstract}
Implantation of a blastocyst into a receptive endometrium is a prerequisite for successful pregnancy. Angiogenesis is a key event in this process but the mechanisms by which localized changes in vascular permeability and angiogenesis occur have yet to be elucidated. Vascular endothelial growth factor (VEGF) and its receptors VEGFR-1 and VEGFR-2 have been implicated as key players in vascular remodelling and placentation. Angiopoietins also appear to have a significant role in regulation of blood vessel growth, maturation and regression. The aim of this study was to describe the molecular regulation of angiogenesis in the first month of pregnancy in marmosets and to address the putative physiological roles for these factors. Uteri were studied at weeks 2, 3 and 4 of pregnancy and compared with late secretory non-pregnant endometrium. Implantation in marmosets occurs at day 11 of pregnancy; hence, these time points were chosen so that the peri-implantation period and very early pregnancy could be studied. mRNAs for VEGF, VEGFR-1 and VEGFR-2, angiopoietin 1, angiopoietin 2 and their receptor Tie-2 were localized and quantified by in situ hybridization. Endothelial cells were
\end{abstract}

identified by CD31 immunocytochemistry. VEGF mRNA was present in all compartments except endothelial cells, and its expression generally increased throughout pregnancy except in upper zone glandular epithelium and luminal epithelium, where a decrease in expression was observed. VEGF receptor mRNAs were found in endothelial cells of the upper zones immediately surrounding glandular epithelium. Angiopoietin 1 mRNA was localized to glandular epithelium of the upper and lower zones throughout pregnancy, and increased in stroma at week 4. Expression of angiopoietin 2 mRNA was localized exclusively to endothelial cells of large luminal vessels and was higher in endometrium from marmosets at week 4 of pregnancy than in endometrium from all other stages. These data provide comprehensive evidence that VEGFR-1 and -2, and angiopoietin 1, angiopoietin 2 and Tie-2 interactions may be involved in the preparation of endometrium for implantation, remodelling of the maternal vasculature and trophoblast invasion during the peri-implantation period in this primate species.

\section{Introduction}

During the last few years, considerable progress has been made in understanding the molecular regulation of angiogenesis in the female reproductive tract. In particular, the role of vascular endothelial growth factor (VEGF) during follicular and luteal development in the marmoset (Callithrix jacchus) model has been established by specific inhibition of this angiogenic factor in vivo (Fraser and Lunn, 2001). As inhibitors of angiogenic factors are likely to be used clinically, it is important

*Correspondence

Email: h.fraser@hrsu.mrc.ac.uk that their role during implantation and early pregnancy is elucidated. The aim of the present study was to build on our knowledge of the regulation of angiogenesis in the reproductive tract of female marmosets by investigating the patterns of expression of angiogenic factors during this period.

Successful pregnancy is dependent on implantation of the blastocyst into the endometrium. Angiogenesis may have a key role in implantation; however, the mechanisms by which localized changes in vascular permeability and angiogenesis occur at the site of implantation have yet to be elucidated.

VEGF and its receptors, fms-like tyrosine kinase (FIt or VEGFR-1) and kinase insert domain-containing region 
(KDR or VEGFR-2) have been implicated as key players in vascular remodelling and placentation in women (Ahmed et al., 1995; Cooper et al., 1995; Clark et al., 1996, 1998; Goldman-Wohl et al., 2000; Helske et al., 2001; Geva et al., 2002; Sugino et al., 2002), sheep (Bogic et al., 2001), pigs (Winther et al., 1999) and marmosets (Wulff et al., 2002) at later stages of pregnancy. VEGF has been localized in endometrium of mice (Chakraborty et al., 1995; Halder et al., 2000), golden hamsters (Yi et al., 1999), rats (Rabbani and Rogers, 2001), rabbits (Das et al., 1997) and Rhesus monkeys (Ghosh et al., 2000) at this time. In mice, inhibition of VEGF prevents oestrogen-induced uterine oedema and implantation (Rockwell et al., 2002), and in rats, the number of implantation sites was reduced after administration of a VEGF blocking antibody (Rabbani and Rogers, 2001).

Angiopoietins also appear to have a major role in regulation of blood vessel growth, maturation and regression, but their importance at the time of implantation has yet to be studied. However, their presence in placenta in later stages of pregnancy has been documented in women (Dunk et al., 2000) and marmosets (Wulff et al., 2002).

The aim of the present study was to describe the localization of mRNA for angiogenic factors and their receptors. Implantation occurs at day 11 after ovulation in marmosets. This study was conducted on tissue collected at the peri-implantation period (week 2 after ovulation) to week 4 of gestation. Angiogenic processes occurring at this early stage of pregnancy are still poorly understood in women and with accumulating knowledge on the effects of manipulation of angiogenesis in marmosets, this species represents a potentially valuable model in which to study these events.

\section{Materials and Methods}

\section{Angiogenesis in marmoset endometrium}

Experiments were carried out under the Animals (Scientific Procedures) Act (1986) and were approved by the local Ethical Review Committee. The tissues studied were collected from marmosets which were the subject of a previous report describing the changes in expression of angiogenic factors associated with early pregnancy in the corpus luteum (Rowe et al., 2002). Blood samples were collected from adult female marmosets three times a week and were assayed for ovulatory increases in plasma progesterone concentration (Wulff et al., 2001). During the mid- to late luteal phase of the second recorded cycle, prostaglandin analogue, $1 \mu \mathrm{g}$ $\mathrm{PGF}_{2 \alpha}$ (cloprostenol; Planate, Coopers Animal Health Ltd, Crewe), was administered i.m. to induce luteolysis so that timing of the subsequent ovulation could be identified accurately. A fertile male was introduced at the time of prostaglandin administration. In anticipation of ovulation occurring 10 days after prostaglandin administration, vaginal lavages were collected on days 7 and 9 of the follicular phase and examined for the presence of spermatozoa. Marmosets were killed humanely at week 2 $(n=4), 3(n=6)$ or $4(n=6)$ after ovulation. A further six non-pregnant late secretory phase animals were also studied (Rowe et al., 2002). Uteri were fixed in $4 \%(\mathrm{w} / \mathrm{v})$ paraformaldehyde (Sigma, Poole).

Pregnancy was confirmed by examining serial sections of the uterus for the presence of a trophoblast and determining plasma concentrations of chorionic gonadotrophin. Chorionic gonadotrophin was measured according to the method of Saltzman et al. (1998). The detection limit for the assay was $12 \mathrm{ng} \mathrm{ml}^{-1}$. All non-pregnant animals had concentrations of CG which were below the detection limit of the assay.

\section{Haematoxylin-eosin staining}

Tissue sections (5 $\mu \mathrm{m}$ thickness) were cut on to Super-frost plus slides (Sigma). Sections were dewaxed in xylene, rehydrated in decreasing concentrations of ethanol, and washed in distilled water before undergoing haematoxylin-eosin staining. The slides were dehydrated and mounted in pertex.

\section{Localization of blood vessels by CD31 immunocytochemistry}

Immunocytochemistry was performed to confirm suggested endothelial cell localization of VEGFR-1, VEGFR-2 and Tie-2 mRNA. Tissue sections (5 $\mu \mathrm{m}$ thickness) were cut on to Super-frost plus slides for immunocytochemistry. The sections were dewaxed in xylene, rehydrated in decreasing concentrations of ethanol and washed in distilled water. Antigen retrieval was performed by boiling sections in a Tefal Clypso pressure cooker in 0.01 mol citrate buffer $\mathrm{I}^{-1}, \mathrm{pH} 6$, for $6 \mathrm{~min}$ at high pressure setting 2 . The slides were then left for 20 min in hot buffer and washed in Tris-buffered saline (TBS:

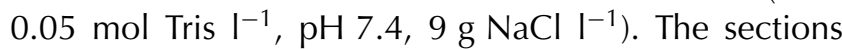
were blocked in normal rabbit serum (diluted 1:5 in TBS) for $30 \mathrm{~min}$ to reduce non-specific binding. The primary antibody CD31 (monoclonal mouse anti-human endothelial cell CD31, Clone JC/70A, no. M0823; Dako, Glostrup) diluted 1:20 in TBS was used. Incubation was performed overnight at $4{ }^{\circ} \mathrm{C}$. The slides were washed three times in TBS. Incubation with the secondary antibody (rabbit anti-mouse Ig; Dako, 1:60 diluted in TBS) was performed for $40 \mathrm{~min}$ at room temperature $\left(22^{\circ} \mathrm{C}\right)$. This was followed by two TBS washes and incubation of the APAAP complex (Dako; 1:100 dilution in normal rabbit serum and TBS) for $40 \mathrm{~min}$ at room temperature. Visualization was performed using nitroblue tetrazolium (NBT) solution containing $45 \mu$ I NBT substrate (Boehringer Mannheim, Mannheim), $10 \mathrm{ml}$ NBT buffer, $35 \mu$ l Xphosphate (Boehringer Mannheim) and $10 \mu$ l levamisole (Sigma). 
Localization of VEGF, VEGFR-1 VEGFR-2, angiopoietin 1, angiopoietin 2 and Tie-2 $m R N A$ in the endometrium

In situ hybridization was performed as described previously using complementary RNA probes for human VEGF, VEGFR-1, VEGFR-2, angiopoietin 1, angiopoietin 2 and Tie-2 (Wulff et al., 2000). The hVEGFR-1 probe was a 641 bp PCR fragment that hybridizes to base pairs 22663 of the Flt mRNA. This comprises the region of the encoding extracellular domain of the Flt protein. Thus, the probe recognizes mRNA for both full-length and soluble Flt. Sense and anti-sense probes were prepared using an RNA transcription kit (Ambion, Austin, TX) and labelled with ${ }^{35}[\mathrm{~S}]$ uridine $5\left[^{\prime}\right]$-triphosphate (NEN, Boston, MA). Deparaffinized sections were treated with $0.1 \mathrm{~mol} \mathrm{HCl} \mathrm{I}^{-1}$ for $20 \mathrm{~min}$ and digested in proteinase $\mathrm{K}\left(5 \mu \mathrm{g} \mathrm{m}{ }^{-1}\right.$; Sigma) for $30 \mathrm{~min}$ at $37^{\circ} \mathrm{C}$. After prehybridization for $2 \mathrm{~h}$ at $55^{\circ} \mathrm{C}$, subsequent hybridization was performed in a moist chamber overnight at $55^{\circ} \mathrm{C}$. High stringency post-hybridization washings and ribonuclease $A$ treatment were used to remove excess probe. The slides were dehydrated, dried and dipped in Ilford G5 liquid emulsion (H. A. West, Edinburgh) and exposed for 12 weeks. The slides were subsequently developed (Kodak D19 developer; Kodak, Rochester, NY) and fixed (Kodak GBS, Kodak). All slides were counterstained with haematoxylin, dehydrated and mounted.

\section{Quantification of in situ hybridization}

Uterine sections were scored and means were calculated according to the grain density in the various different uterine compartments: namely, luminal epithelium, upper zone stroma (area beneath the luminal epithelium), upper zone glands, lower zone glands (area adjacent to myometrium), lower zone stroma and endothelial cells. Scoring was performed by two independent observers with the following classification: - : no detectable specific signal above background levels; + (1): detectable but low grain density clearly above background levels; $++(2)$ : moderate grain density; $+++(3)$ : high grain density without grain coalescence; and ++++ (4): grain coalescence. Scoring of uteri was blind and random by each observer. An overall score was assigned for each compartment within a section. Significant differences between groups $(P<0.05)$ were determined using a Kruskal-Wallis non-parametric test followed by Dunn's multiple comparisons test. Representative photographs illustrate the localization of mRNAs. In general, these reflect the quantitative scoring but also show focal changes within a compartment not distinguished by the scoring.

\section{Results}

\section{Vascular endothelial growth factor (VEGF)}

VEGF mRNA was localized to the glandular epithelium in the late secretory phase non-pregnant endomet- rium. Highest grain density was observed in the luminal epithelium and glands of the upper zones. Grain density was much reduced in the mid-zone glands, whereas grains were completely absent from the lower zone (Fig. 1a,b). Exact cellular localization of VEGF mRNA to glandular epithelium was confirmed under high power images (Fig. 1j).

In early pregnant endometrium, at 2 weeks after ovulation, a similar localization was observed to that in the non-pregnant animals (Fig. 1c-e). However, low expression of stromal VEGF mRNA was apparent just beneath the luminal epithelium (Fig. 1c,d). At this very early stage of pregnancy, 3 days after implantation, no expression of VEGF mRNA was observed in the blastocyst or fetal membranes (Fig. 1c,d). By week 3 of pregnancy (day 21), expression of VEGF mRNA remained high in the glandular epithelium. Lower expression was also observed in the lower zone glands (Fig. 1h,k). In decidua, VEGF expression was relatively high and punctate (Fig. 1f,g,i). In addition, by this stage of pregnancy the blastocyst and fetal membranes were expressing VEGF mRNA. Animals at week 4 of pregnancy (day 28) had similar VEGF mRNA localization to animals at week 3; hence, a representative figure at 3 weeks is shown (Fig. 1f (dark field) and Fig. 1g (bright field). In all sense slides, no specific signal was detected above a low background (Fig. $1 \mathrm{~h}$ insert).

\section{KDR and Flt (VEGFR-1 and VEGFR-2)}

VEGFR-2 mRNA localization is shown (Fig. 2). It should be noted that expression of VEGFR-1 mRNA was consistently lower than that of VEGFR-2, but it was found in the same cellular locations; thus, mainly photographs of VEGFR-2 mRNA are shown (Fig. 2a-l). However, representative images are included of VEGFR-1 hybridization at week 2 of pregnancy (Fig. 2m,n). Each receptor localization and grain density was scored separately (Table 1 ). In the late secretory non-pregnant endometrium, VEGFR-2 mRNA was found exclusively in endothelial cells surrounding the upper zone glands beneath the luminal epithelium (Fig. 2a,b,j). This observation was confirmed by comparison with CD31 immunocytochemistry to identify endothelial cells in sequential sections (Fig. 2C). In endometrium from week 2 of pregnancy, VEGFR-2 mRNA was localized to the upper zone endothelium as in non-pregnant endometrium. Grains were absent from the blastocyst and fetal membranes (Fig. 2d,e). At weeks 3 and 4 of pregnancy, expression of VEGFR-2 mRNA remained in endothelial cells of the upper zone with highest grain density observed around large maternal vessels beneath the luminal epithelium (Fig. 2g,h,k), which was again confirmed by comparison with CD31 localization (Fig. 2i). VEGFR-2 mRNA was also observed to a lesser extent throughout the stromal compartment, again localized to endothelial cells (Fig. 2l). In all sense slides, no specific 

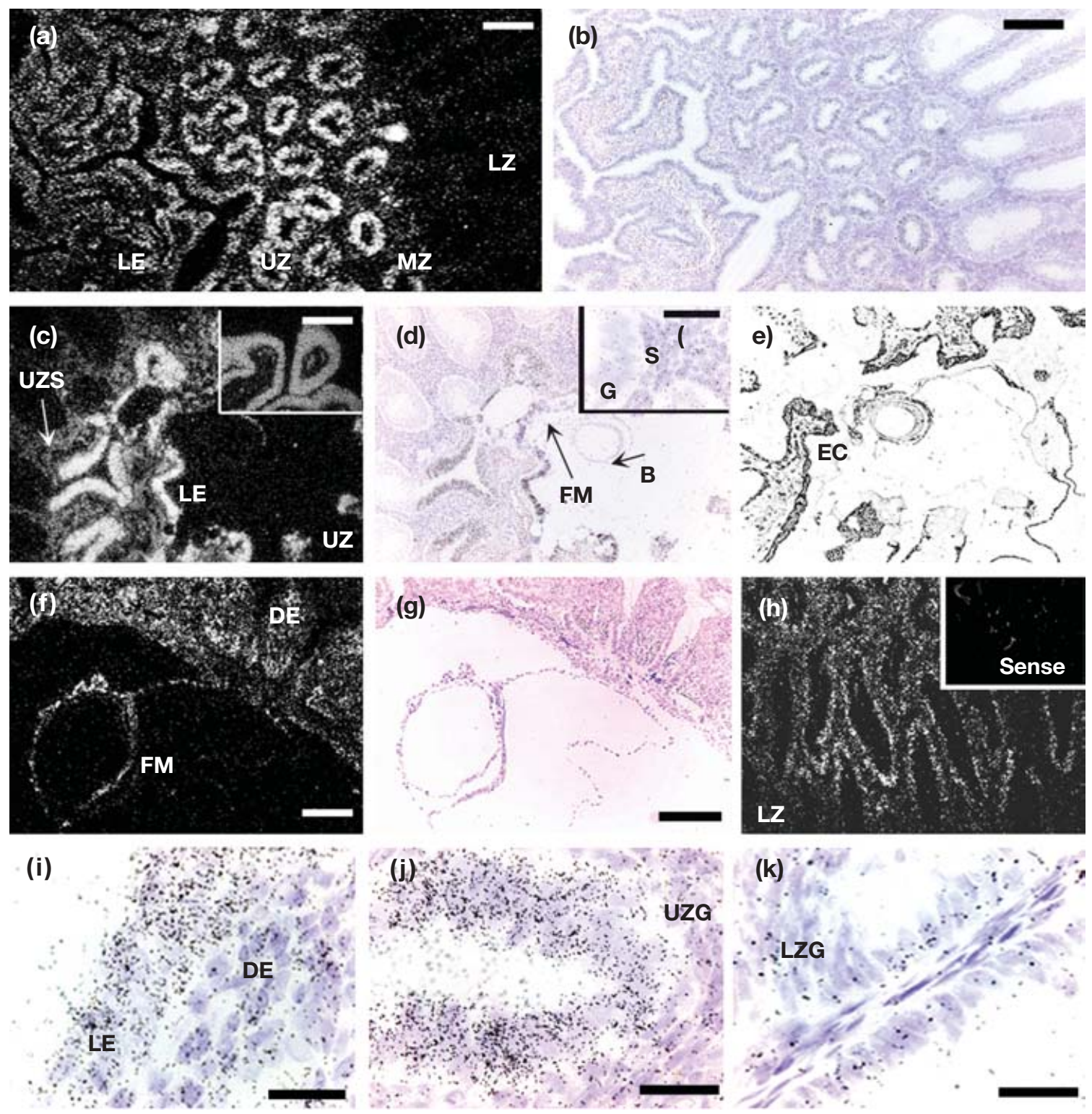

Fig. 1. (a-k) In situ hybridization of vascular endothelial growth factor (VEGF) mRNA in marmoset (Callithrix jacchus) endometrium. $(\mathrm{a}, \mathrm{c}, \mathrm{f})$ Dark-field and $(\mathrm{b}, \mathrm{d}, \mathrm{g})$ corresponding light-field images. $(\mathrm{a}, \mathrm{b})$ Non-pregnant late secretory phase endometrium. Note high expression in upper zone glands (UZ) and luminal epithelium (LE), which decreases in the mid-zone $(\mathrm{MZ})$ and is absent in the lower zone (LZ). (c,d,e) Endometrium from pregnant marmoset at day 14 after ovulation. (c,d) Note high expression in luminal epithelium (LE) and upper zone (UZ) glands, and absence of expression in the blastocyst (B) and fetal membranes (FM). Lower VEGF expression can be seen in the upper zone stroma (UZS) in (c). Insert in (c) is a higher power image of the luminal epithelium $(\times 40)$. Insert in (d) is a higher power bright-field image of the upper zone stroma $(\times 100)$. (e) CD31 staining for endothelial cells (EC) to emphasize non-endothelial localization of stromal VEGF. (f-h) Typical VEGF expression at day 21 and day 28. (f) Dark-field image of marmoset early implantation site. Note high punctate expression in the decidualized endometrium (DE) and appearance of expression in the fetal membranes (FM). (g) Corresponding light-field image to (f). (h) Dark-field image of lower zone (LZ) endometrium where there is low glandular VEGF expression. Insert in (h) is a representative sense photograph. (i) Higher power $(\times 100)$ bright-field image of the luminal epithelium (LE) of an animal at week 3 of pregnancy; this image also illustrates the hybridization observed in decidualized stroma (DE). (j) High power $(\times 100)$ image of the upper zone glands (UZG) in late secretory non-pregnant endometrium to confirm cellular localization. (k) High power $(\times 100)$ image to highlight expression of VEGF in the lower zone glands (LZG) at this time. G: gland; S: stroma. Scale bars represent (a,b,f,g) 100, (c,d inserts) 50 and (i-k) $25 \mu \mathrm{m}$. 

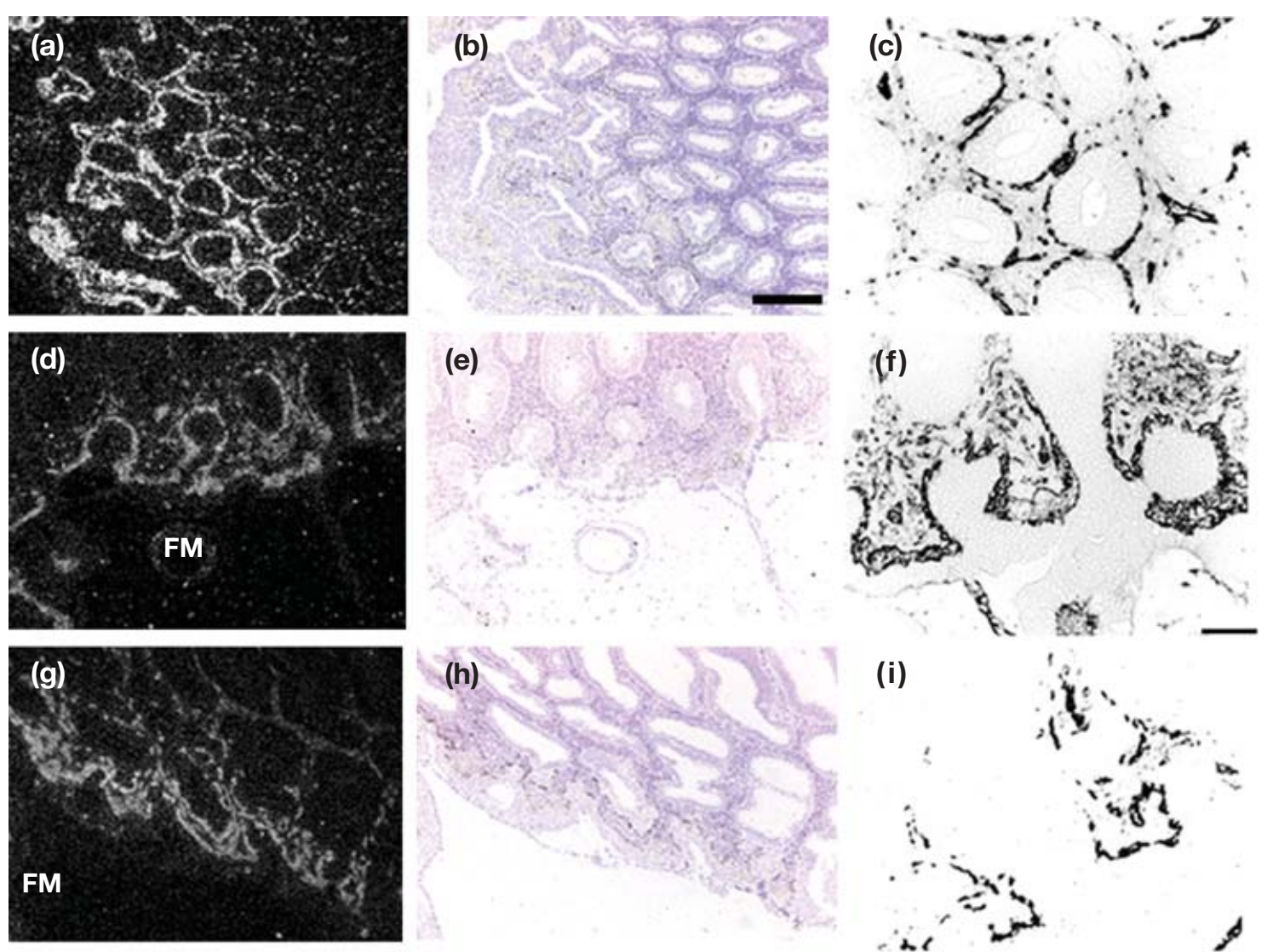

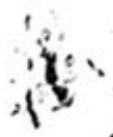
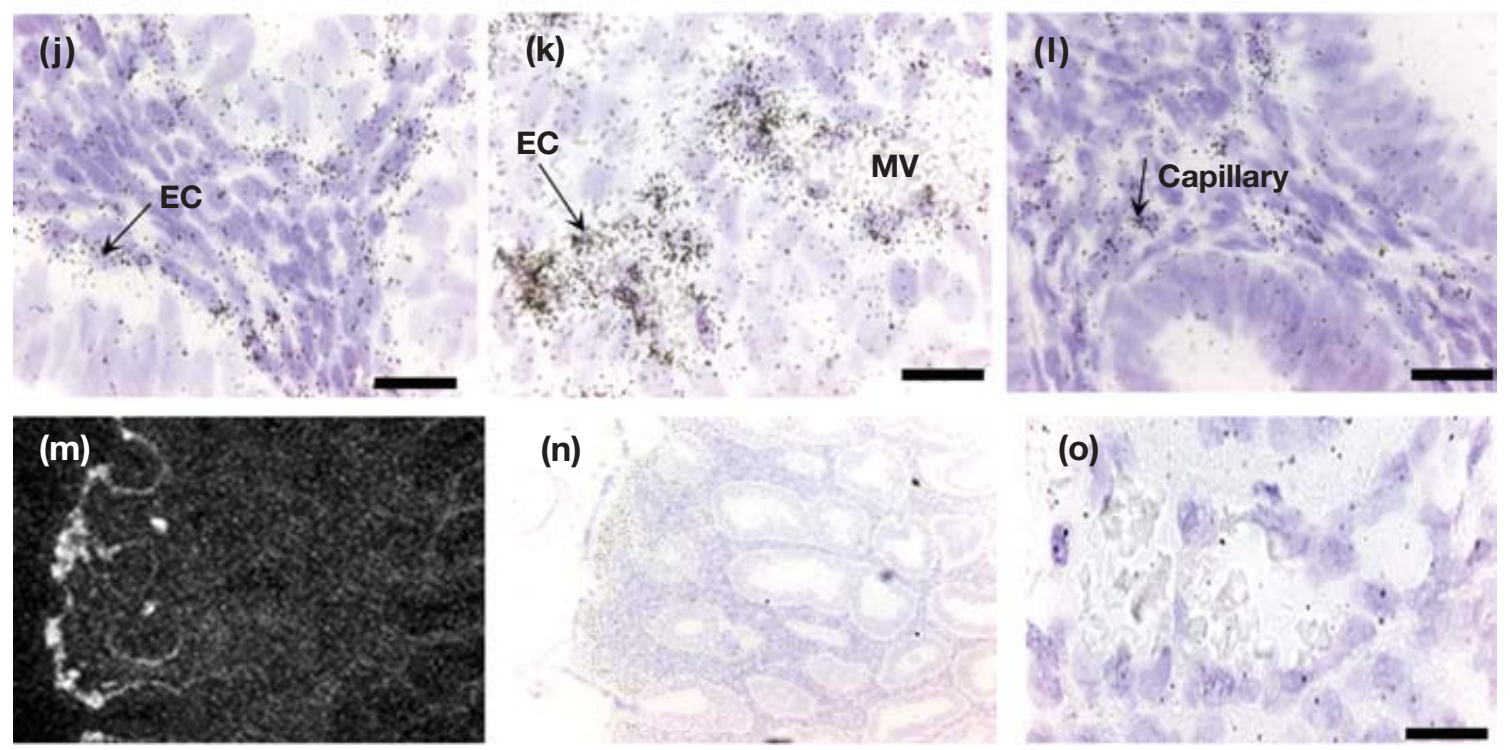

Fig. 2 In situ hybridization of vascular endothelial growth factor receptor (VEGFR) (a-l) -2 and (m,n) -1 mRNA in marmoset (Callithrix jacchus) endometrium. (a-c) Non-pregnant late secretory phase endometrium. (a) Dark-field and (b) light-field images of VEGFR-2 expression in upper zone endometrium. Note endothelial localization immediately surrounding glands as demonstrated by (c) CD31 immunocytochemistry and (j) high power $(\times 100)$ bright-field image. Also note punctate expression in endothelial cells throughout the stroma in all stages studied illustrated by a representative high power bright-field image (I). (d-f) Pregnant marmoset endometrium at day 14 after ovulation. (d) Dark-field and (e) corresponding light-field images showing localization to large and small luminal blood vessels surrounding the uppermost glands as indicated by CD31 immunocytochemistry for endothelial cells (f). Note absence of grains in blastocyst and fetal membranes (FM). (g) Dark-field and (h) light-field images of VEGFR-2 expression at 3-4 weeks. Note localization in upper zone to luminal maternal vessels (MV) as demonstrated by CD31 immunocytochemistry (i), and high power bright-field image $(\mathrm{k})$. In addition, note absence of localization to fetal membranes $(\mathrm{g})$ and punctate throughout the stroma of lower zones (I). (o) A high power ( $\times 100)$ bright-field sense image. Scale bars represent (b) 100, (f,i) 50 and $(\mathrm{j}-\mathrm{l}, \mathrm{o}) 25 \mu \mathrm{m}$. 
Table 1. Quantification of in situ hybridization using relative grain density within uterine compartments of marmosets (Callithrix jacchus) throughout the stages of pregnancy studied for vascular endothelial growth factor (VEGF), VEGFR-1 and -2, angiopoietin 1, angiopoietin 2 and Tie-2

\begin{tabular}{|c|c|c|c|c|c|c|}
\hline $\begin{array}{l}\text { Stage of cycle } \\
\text { of endometrium }\end{array}$ & $\begin{array}{l}\text { Luminal } \\
\text { epithelium }\end{array}$ & $\begin{array}{l}\text { Upper zone } \\
\text { glandular } \\
\text { epithelium }\end{array}$ & $\begin{array}{l}\text { Upper zone } \\
\text { stroma }\end{array}$ & $\begin{array}{l}\text { Upper zone } \\
\text { endothelium }\end{array}$ & $\begin{array}{l}\text { Lower zone } \\
\text { glandular } \\
\text { epithelium }\end{array}$ & $\begin{array}{l}\text { Lower zone } \\
\text { stroma }\end{array}$ \\
\hline \multicolumn{7}{|l|}{ VEGF } \\
\hline Late secretory, non-pregnant & $+++^{\mathrm{a}}$ & +++ & - & na & - & - \\
\hline Week 2 of pregnancy & $+++^{\mathrm{ab}}$ & ++ & + & na & - & - \\
\hline Week 3 of pregnancy & $+^{\mathrm{ab}}$ & ++ & ++ & na & + & - \\
\hline Week 4 of pregnancy & $-{ }^{\mathrm{b}}$ & + & ++ & na & ++ & - \\
\hline \multicolumn{7}{|l|}{ VEGFR-2 (-1) } \\
\hline Late secretory, non-pregnant & - & - & - & $+++(+)$ & - & - \\
\hline Week 2 of pregnancy & - & - & - & $++(+)$ & - & - \\
\hline Week 3 of pregnancy & - & - & - & $++(+)$ & - & - \\
\hline Week 4 of pregnancy & - & - & - & $++(+)$ & - & - \\
\hline \multicolumn{7}{|l|}{ Angiopoietin 1} \\
\hline Late secretory, non-pregnant & - & + & + & na & - & - \\
\hline Week 2 of pregnancy & - & + & ++ & na & +++ & - \\
\hline Week 3 of pregnancy & - & + & +++ & na & ++ & - \\
\hline Week 4 of pregnancy & - & + & +++ & na & + & - \\
\hline \multicolumn{7}{|l|}{ Angiopoietin 2} \\
\hline Late secretory, non-pregnant & - & - & - & $-^{\mathrm{a}}$ & - & - \\
\hline Week 2 of pregnancy & - & - & - & $-^{\mathrm{a}}$ & - & - \\
\hline Week 3 of pregnancy & - & - & - & $-{ }^{\mathrm{a}}$ & - & - \\
\hline Week 4 of pregnancy & - & - & - & $+++{ }^{\mathrm{b}}$ & - & - \\
\hline \multicolumn{7}{|l|}{ Tie-2 } \\
\hline Late secretory, non-pregnant & - & - & + & +++ & - & + \\
\hline Week 2 of pregnancy & - & - & + & ++ & - & - \\
\hline Week 3 of pregnancy & - & - & + & ++ & - & + \\
\hline Week 4 of pregnancy & - & - & + & ++ & - & + \\
\hline
\end{tabular}

Analysis was performed by two independent observers. A Kruskal-Wallis test followed by a Dunn's multiple comparison test was used to determine significant differences within a particular uterine compartment between groups.

Values given are mean scores. na: not assessed.

${ }^{\mathrm{ab}}$ Different letters denote significant differences $(P<0.05)$. No statistical comparisons were made between uterine compartments within the same stage of the cycle.

signal was detected above a low background. This is shown in (Fig. 20), a $\times 100$ magnification bright-field image of the corresponding sense slide to Fig. $2 \mathrm{k}$.

\section{Angiopoietin 1}

Angiopoietin $1 \mathrm{mRNA}$ was localized to upper zone stroma and, to a lesser extent, the glandular epithelium of the upper zone in late secretory non-pregnant endometrium. In pregnant endometrium at day 14 , there was high expression of angiopoietin $1 \mathrm{mRNA}$ in the lower zone glands (Fig. 3d,e,o) but it was completely absent from adjacent compartments, the myometrium and midzone endometrium (see insert Fig. 3d). In the upper zone, expression of angiopoietin $1 \mathrm{mRNA}$ was low in the glandular epithelium but much more intense in stroma (Fig. 3p). Grains were absent from blastocyst and fetal membranes (Fig. 3f,g). At week 3 of pregnancy, upper and lower zone glandular epithelial expression of angiopoietin 1 mRNA was retained (Fig. 3h,j). Grain density increased in the stroma at this stage (Table 1; Fig. 3h,i). By day 28, clear, punctate angiopoietin 1 mRNA localization was observed throughout the decidualized stroma (Fig. $3 k, I, n$ ). In all sense slides, no specific signal was detected above a low background.

\section{Angiopoietin 2}

Angiopoietin 2 mRNA was detected only in animals at week 4 of pregnancy, and it was localized exclusively 
in endothelial cells of maternal vessels (Table 1; Fig. 4). In all sense slides, no specific signal was detected above a low background.

\section{Tie-2}

In non-pregnant late secretory endometrium Tie-2 mRNA was expressed predominantly in endothelial cells throughout the stroma of all zones (Fig. 5a,b,l). At week 2 of pregnancy (day 14), the generalized pattern of stromal expression observed in the non-pregnant animals was reduced but was still present (Table 1; Fig. 5d,e). High grain density was observed in endothelium immediately surrounding the upper zone glands at this time (Fig. 5d,e,j). By weeks 3 and 4 of pregnancy, luminal epithelial expression of Tie-2 mRNA was not observed but expression was still observed in upper zone decidualized stroma (Fig. 5g). By comparison with CD31 immunocytochemistry (Fig. 5i) and high power haematoxylin-eosin-stained sections (insert Fig. 5h), Tie-2 appeared to be expressed in blood vessels throughout the decidua. In all sense slides, no specific signal was detected above a low background.

\section{Discussion}

In the present study the spatial and temporal patterns of expression of mRNAs for VEGF, angiopoietins and their receptors in marmoset uterus were described during the peri-implantation period, allowing us to begin to construct a working hypothesis as to their potential role in oxygen and nutrient exchange and maternal vascular remodelling.

Implantation occurs on day 11 after ovulation in marmosets, 3 days later than in women, and the blastocyst remains in loose attachment to the surface epithelium without epithelial fusion. Penetration of trophoblast into maternal vessels occurs quite late, at days 45-60 of gestation, compared with about day 12 in women (Enders, 1993). Therefore, fetal demand for nutrients and oxygen during early pregnancy must be met by diffusion across the fetomaternal interface, a process likely to be critically dependent on vascular permeability. Increased permeability is generally thought to accompany tissue growth and remodelling (Dvorak et al., 1999; Dvorak, 2000; Rabbani and Rogers, 2001). VEGF is a vascular permeability factor as well as a mitogen for endothelial cells (Keck et al., 1989). Permeability and uterine oedema mediated by VEGF are critical for successful implantation in rodents, in which inhibition of VEGF prevented implantation (Dvorak et al., 1999; Dvorak, 2000; Rabbani and Rogers, 2001). Our finding that VEGF mRNA was localized to upper zone endometrium of all groups, increasing during the first few weeks of pregnancy, implies a role for VEGF as a permeability factor at this time.

The presence of VEGF mRNA in upper zone glandular epithelium and luminal epithelium of non-pregnant late secretory endometrium, with mRNA for both VEGFR-1 and -2 in endothelial cells of blood vessels immediately surrounding these glands, is indicative of a local action of VEGF. This is also observed in endometrium at weeks 2 and 3 of pregnancy where it may be involved in promoting fenestration of these vessels, thereby facilitating oxygen transfer to the avascular fetus. Although fetal membranes positive for CD31 were observed, they had no capillary formation. It may be that these cells are destined to become differentiated endothelial cells and thus already express CD31 surface antigens.

Upregulation of stromal VEGF mRNA was observed at week 4 of pregnancy. It is likely that the permeability role of VEGF is decreasing at this point, as glandular secretions are reduced and maternal sinusoids, although not yet invaded by trophoblast, have much more intimate contact with the more closely attached blastocyst, thus facilitating diffusion. Very strong expression of VEGFR-1 and -2 mRNA was also observed in endothelial cells of large maternal vessels immediately beneath the luminal epithelium where fetal VEGF could be acting to promote vessel migration such that fetal metabolic demands can be adequately met. The pattern of mRNA localization of VEGF and its receptors observed in the present study is consistent with findings in women, in whom fetal VEGF expression in tripronuclear zygotes has been implicated to induce neoangiogenesis at the site of implantation (Krussel et al., 2000). VEGF expression has been documented in human placenta and in other species in the later stages of placentation where it is expressed in maternal decidua, but unlike our findings, its receptor VEGFR-1 appeared to be expressed in invading syncytial trophoblast (Ahmed et al., 1995; Cooper et al., 1995; Clark et al., 1996). It may be that fetal VEGF receptors are present only at later stages in marmosets, when a more intimate interplay between maternal and fetal tissues occurs (Wulff et al., 2002). Soluble Flt has been reported to be produced by human trophoblast in pregnancy and is detectable in the serum of pregnant women (Banks et al., 1998). It is possible that this also occurs in marmosets; however, no hybridization of the VEGFR-1 probe to trophoblast was observed in the present study despite our probe detecting both the soluble and full-length receptor. No serum analyses were conducted.

The roles of the angiopoietins and their receptor Tie-2 in early pregnancy are just beginning to be explored. In general, angiopoietin 1 acts on its receptor Tie- 2 to promote stabilization of the endothelial cell-vascular smooth muscle structure (Suri et al., 1996), whereas angiopoietin 2 acts as an antagonist by binding to the Tie-2 receptor without transmitting a signal (Maisonpierre et al., 1997; Lobov et al., 2002). In marmoset uterus, 

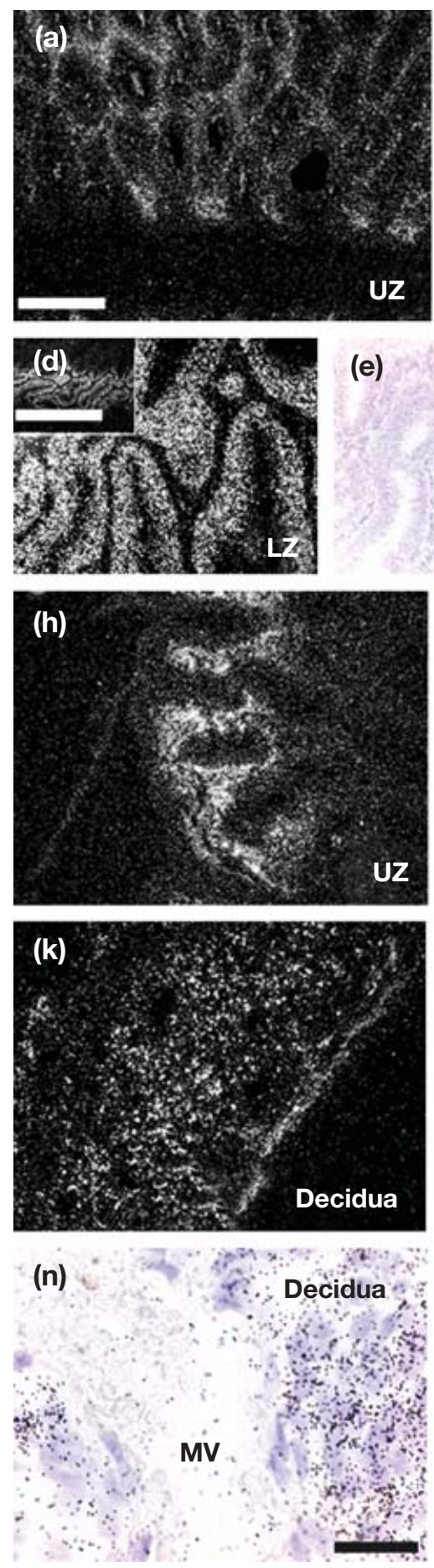

(e)
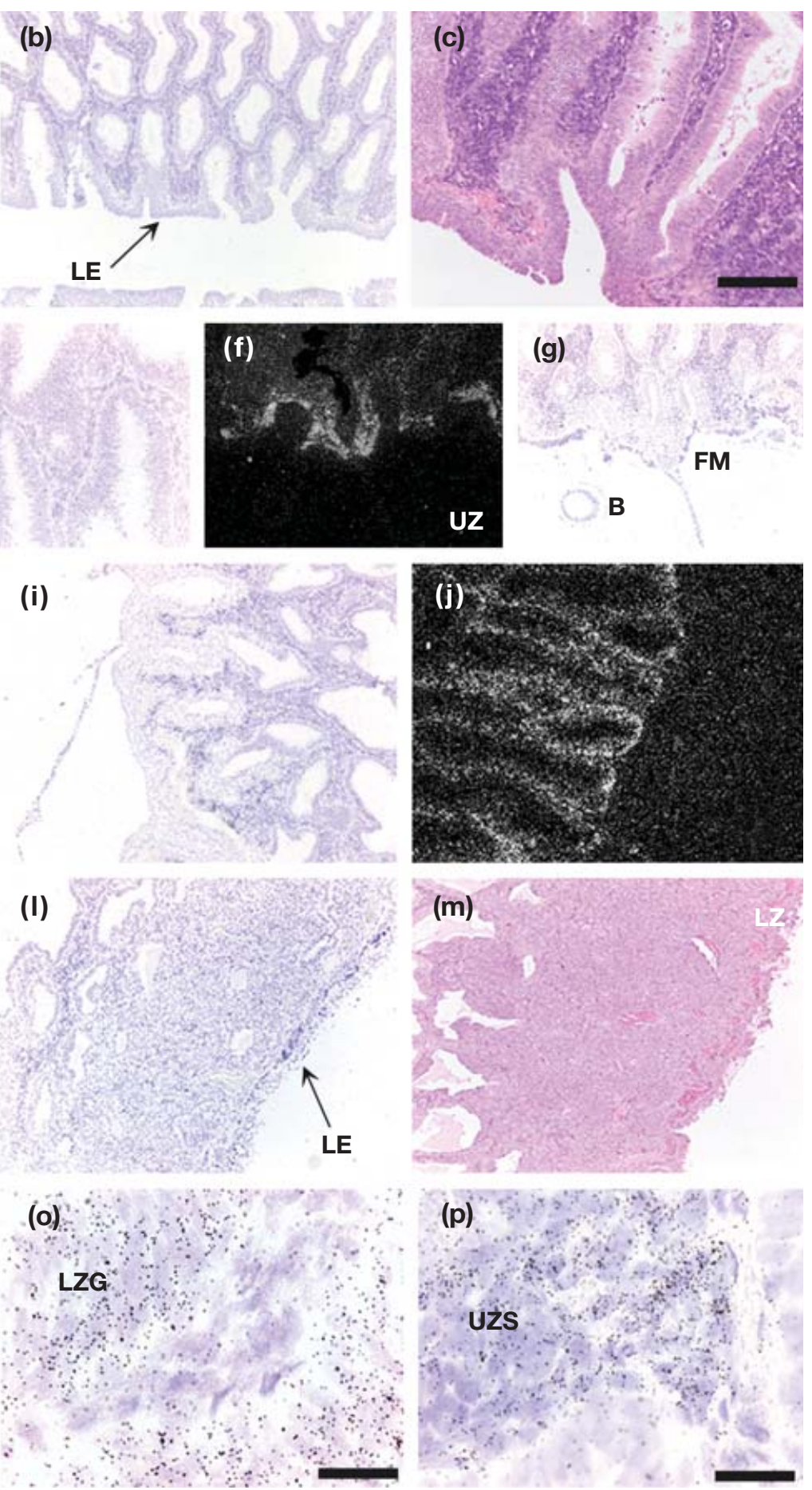
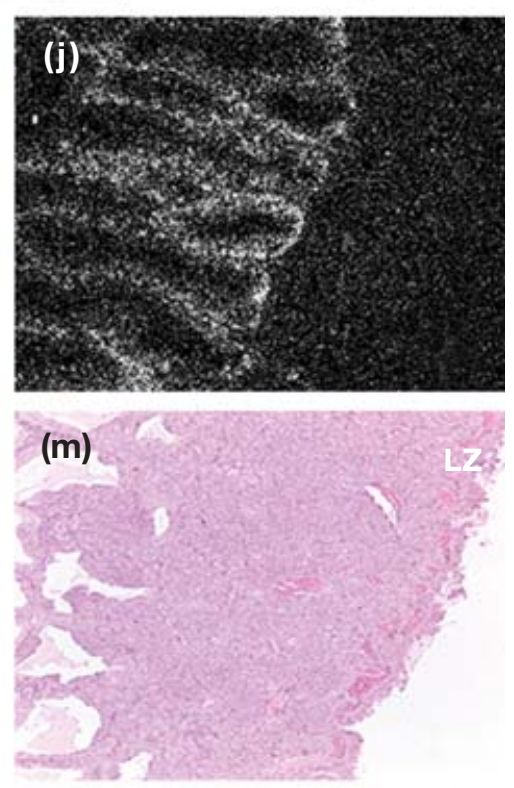

(p)

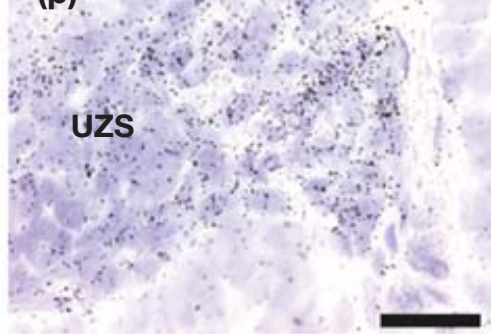

Fig. 3 (a-p) In situ hybridization of angiopoietin 1 mRNA in marmoset (Callithrix jacchus) endometrium. (a) Dark-field and (b) corresponding light-field images of non-pregnant late secretory phase endometrium. Note angiopoietin 1 expression in stroma and low expression in glands of the upper zone endometrium. LE: luminal epithelium. (c) Haematoxylin-eosinstained section of upper zone and luminal epithelium $(\times 40)$. $(\mathrm{d}-\mathrm{g})$ Day 14 pregnant marmoset endometrium. (d) Dark-field and (e) corresponding light-field images of high expression of angiopoietin $1 \mathrm{mRNA}$ in the glandular epithelium of the lower zone (LZ). Insert in (d) is a low power image $(\times 10)$ to demonstrate absence of grains in myometrium and mid-zone endometrium. (o) High power image $(\times 100)$ to confirm cellular localization to glands of the lower zone (LZG). (f) Dark-field and (g) corresponding light-field images of angiopoietin 1 localization in the upper zone (UZ). Note low glandular localization together with stromal expression. Angiopoietin 1 is absent from the fetal membranes (FM) and blastocyst (B). (h-j) Angiopoietin 1 expression at day 21 of pregnancy. (h) Dark-field and (i) light-field images of 
angiopoietin 1 mRNA was the most abundantly expressed angiopoietin localized to upper zone glands and stroma, whereas Tie-2 mRNA was expressed in endothelial cells. In menstruating species after an infertile cycle, menstruation and shedding of the upper zones of the endometrium occur. The lower zone and myometrium are comparatively more stable compartments from which a new vascular and new upper zone endometrium is supplied (Smith, 2001). Although the marmoset does not menstruate, in infertile cycles a degree of vascular remodelling may occur in the upper zones, whereas the lower zones remain more stable, implicating a role for angiopoietin 1 in this region as was observed in the present study. However, the fertility rate is extremely high in marmosets (Rowe et al., 2002) and hence this species may retain a stable upper zone endometrium without remodelling in response to progesterone withdrawal, associated with basal expression of angiopoietin 1 and Tie-2 in preparation for pregnancy. In fertile cycles, the endometrium is preparing to receive an implanting blastocyst and, thus, would require a stable maternal vasculature. It is possible that angiopoietin 1 mRNA may be expressed in the lower zones to maintain core vessel stability.

During the peri-implantation period, stromal angiopoietin 1 and Tie-2 mRNA expression in the upper zones adjacent to the site of implantation was increased compared with non-pregnant endometrium. As gestation progressed into weeks 3 and 4, expression of angiopoietin 1 and Tie- 2 mRNA in upper zone stroma and decidua was further increased. This corresponds with a continued requirement for a stable vasculature at the maternal-fetal interface and eventual placentation and development of a haemochorial placenta. This role appears to extend to later pregnancy in marmosets, in which angiopoietin $1 \mathrm{mRNA}$ is localized exclusively to syncytial trophoblast (Wulff et al., 2002), indicating that once the fetal vasculature begins to develop, there may be a need for stabilization of newly formed fetal vessels.

The only site of localization of angiopoietin 2 mRNA was in large maternal vessels immediately beneath the luminal epithelium of animals at week 4 of pregnancy, where it was co-localized with Tie-2 mRNA. This is similar to the situation observed in later stages of placentation in marmosets (Wulff et al., 2002). The presence of VEGF mRNA in the decidualized upper zone endometrium with its receptors in endothelial cells of this same region, including large maternal vessels, is indic-
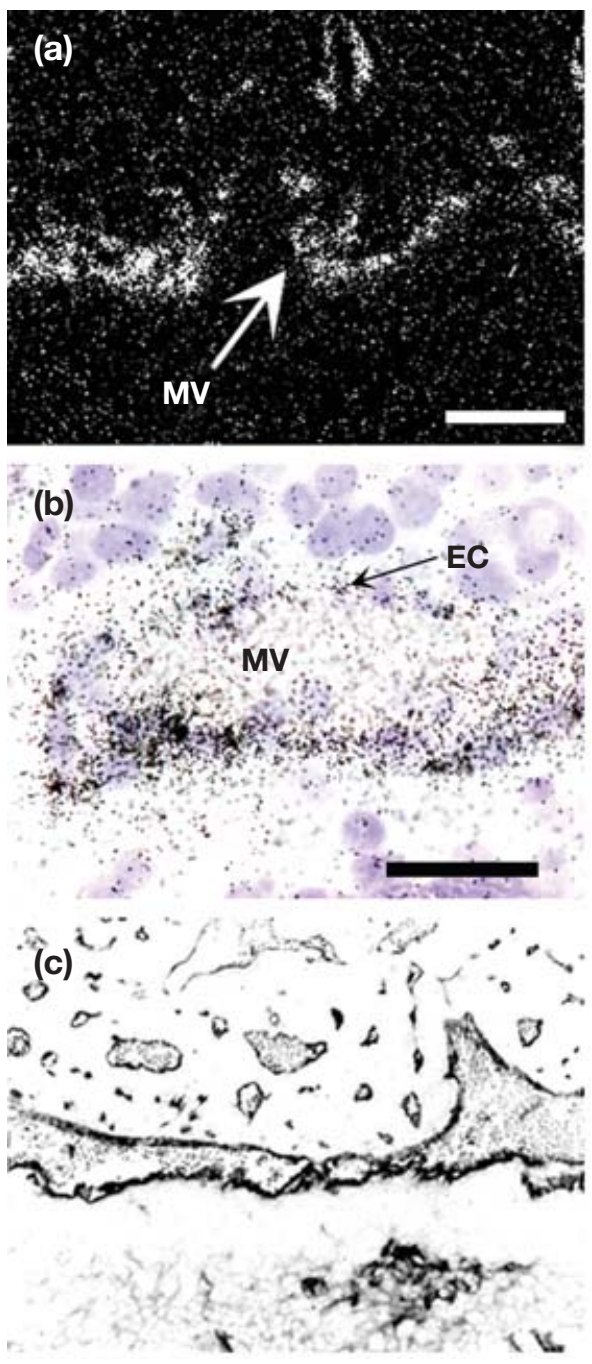

Fig. $4(\mathrm{a}-\mathrm{C})$ In situ hybridization of angiopoietin 2 mRNA in pregnant marmoset (Callithrix jacchus) endometrium at day 28. (a) Dark-field $(\times 20)$ and (b) light-field $(\times 100)$ images of upper zone endometrium. Note angiopoietin 2 expression in endothelial cells (EC) of large maternal vessels (MV), as demonstrated by CD31 immunocytochemistry in (c) and higher power image in (b). Angiopoietin 2 was absent from all other stages studied. Scale bars represent (a) 100 and (b) $25 \mu \mathrm{m}$.

ative of a similar paracrine modulation. It is thought that angiopoietin 2, when expressed in conjunction with VEGF, can be pro-angiogenic, promoting destabilization of vessels and breakdown of basal lamina to allow migration of endothelial cells and subsequent angiogenesis.

angiopoietin 1 expression in the upper zone. Note expression in glandular epithelium and to a greater extent in stroma. This is also highlighted in $(p)$, a high power $(\times 100)$ bright-field image. UZS: upper zone stroma. (j) Dark-field image of lower zone (LZ) endometrium. Note glandular epithelial localization of angiopoietin 1. (k-m) Angiopoietin 1 expression at day 28 of pregnancy. (k) Dark-field and (I) corresponding light-field images of decidualized endometrium and luminal epithelium (LE). Note punctate expression of angiopoietin 1 mRNA throughout decidualized stroma, not in endothelial cells, which is further illustrated in $(\mathrm{n})$, a higher power $(\times 100)$ bright-field image. MV: maternal vessel. $(\mathrm{m})$ Haematoxylin-eosin-stained section to demonstrate decidual morphology. Scale bars represent (a) 100, (c) 50, (d insert) 200 and (n-p) $25 \mu \mathrm{m}$. 

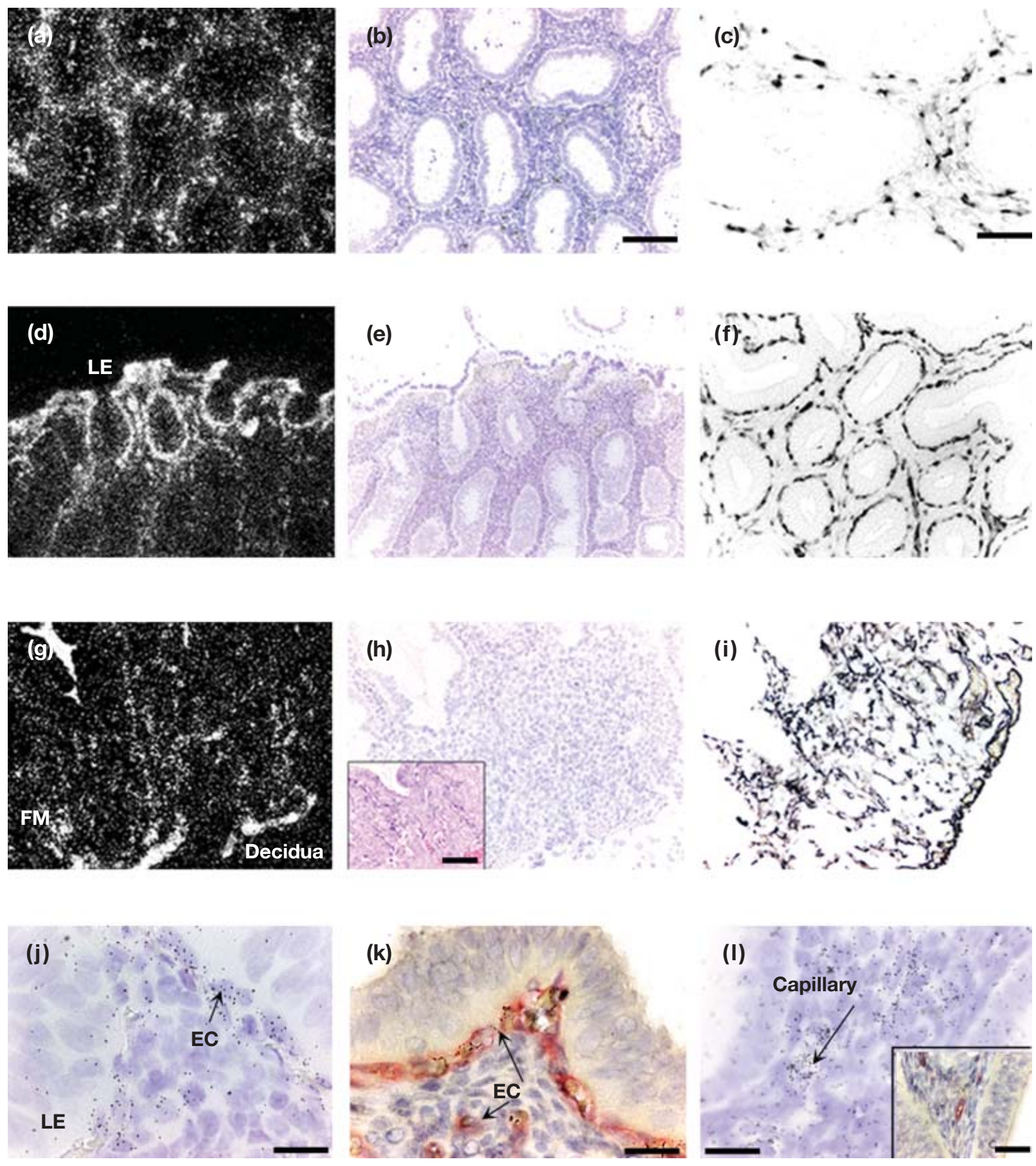

Fig. 5. (a-l) In situ hybridization of Tie-2 mRNA in marmoset (Callithrix jacchus) endometrium. (a-c) Non-pregnant late secretory phase endometrium. (a) Dark-field and (b) light-field images of Tie-2 expression in stroma throughout all zones of the nonpregnant endometrium. Note expression localized to endothelial cells as demonstrated by CD31 immunocytochemistry (c) taken at $\times 40$ objective and higher power $(\times 100)$ bright-field images in (I). The insert in (I) illustrates CD31 immunocytochemistry, counterstained with haematoxylin. ( $\mathrm{d}-\mathrm{f}$ ) Pregnant marmoset endometrium at day 14 after ovulation. (d) Dark-field and (e) corresponding light-field images of upper zone endometrium. Note localization to endothelial cells (EC) immediately surrounding upper zone glands as demonstrated by (f) CD31 immunocytochemistry and (j,k) higher power (x 100) brightfield images. (k) CD31 immunocytochemistry counterstained with haematoxylin. LE: luminal epithelium. (g-i) Typical Tie-2 expression at day 21 and day 28 of pregnancy. (g) Dark-field and (h) light-field images of upper zone decidualized endometrium. FM: fetal membranes Note punctate expression of Tie-2 probably localized to endothelial cells as demonstrated by (i) CD31 immunocytochemistry. Insert shows a haematoxylin-eosin-stained section. Note vessels similar to areas of grain localization in (g). Scale bars represent (b) 100, (c,h insert) 50 and (j-l, I insert) $25 \mu \mathrm{m}$. 
Our findings indicate that at week 4 of pregnancy, VEGF and angiopoietin 2 may be working synergistically to promote migration of these vessels and establishment of an efficient interface for nutrient and oxygen exchange between the fetal and maternal circulation.

Neither angiopoietin nor Tie-2 mRNA was detected in blastocyst or fetal membranes. Their presence in first trimester placenta has been reported in women; Tie-2 has also been localized to trophoblast cells, as well as endothelial cells (Dunk et al., 2000). However, Tie-2 mRNA has been observed in trophoblast in later stages of pregnancy in marmosets (Wulff et al., 2002).

It is also of interest that angiopoietin 2 is expressed by uterine natural killer cells in human endometrium, particularly those situated beneath the luminal epithelium and around vascular smooth muscle cells (Smith, 2001). No evidence for such expression was observed in marmosets, in which angiopoietin 2 mRNA was found exclusively in endothelial cells of luminal maternal vessels as confirmed by CD31 immunocytochemistry.

In conclusion, this study has described the molecular regulation of angiogenesis associated with implantation and vascular remodelling during the peri-implantation period in marmosets. It provides evidence that VEGFR1 and -2 and angiopoietin 1, angiopoietin 2 and Tie-2 interactions may be involved in the preparation of endometrium for implantation, remodelling of the maternal vasculature during the peri-implantation period and trophoblast invasion. Taken together with our previous report on the molecular angiogenic events occurring from week 7 of pregnancy to term, a comprehensive picture of implantation and placentation in this primate species is provided. Having proposed putative roles for these angiogenic regulators in this process, there is potential for in vivo studies using specific antagonists to determine their physiological function.

The authors would like to thank Regeneron Pharmaceuticals, Inc., for the gift of angiopoietin and Tie-2 cDNA probes; S. Charnock-Jones for the gifts of VEGF, VEGFR-1 and VEGFR-2 cDNA probes; I. Swanston and F. Pitt for assays; and J. Roser, A. L. Parlow and NIDDK's National Hormone and pituitary Program for reagents for the CG assay.

\section{References}

Ahmed A, Li XF, Dunk C, Whittle MJ, Rushton DI and Rollason T (1995) Colocalisation of vascular endothelial growth factor and its flt-1 receptor in human placenta Growth Factors 12 235-243

Banks RE, Forbes MA, Searles J et al. (1998) Evidence for the existence of a novel pregnancy-associated soluble variant of the vascular endothelial growth factor receptor, Flt-1 Molecular Human Reproduction 4 377-386

Bogic LV, Brace RA and Cheung CY (2001) Developmental expression of vascular endothelial growth factor (VEGF) receptors and VEGF binding in ovine placenta and fetal membranes Placenta 22 265-275

Chakraborty I, Das SK and Dey SK (1995) Differential expression of vascular endothelial growth factor and its receptor mRNAs in the mouse uterus around the time of implantation Journal of Endocrinology 147339 352
Clark DE, Smith SK, Sharkey AM and Charnock-Jones DS (1996) Localization of VEGF and expression of its receptors flt and KDR in human placenta throughout pregnancy Human Reproduction $\mathbf{1 1}$ 1090-1098

Clark DE, Smith SK, Licence D, Evans AL and Charnock-Jones DW (1998) Comparison of expression patterns for placenta growth factor, vascular endothelial growth factor (VEGF), VEGF-B and VEGF-C in human placenta throughout gestation Journal of Endocrinology 159 459-467

Cooper JC, Sharkey AM, Mclaren J, Charnock-Jones DS and Smith SK (1995) Localization of vascular endothelial growth factor and its receptor, flt, in human placenta and decidua by immunohistochemistry Journal of Reproduction and Fertility 105 205-213

Das SK, Chakraborty I, Wang J, Dey SK and Hoffman LH (1997) Expression of vascular endothelial growth factor (VEGF) and VEGFreceptor messenger ribonucleic acids in the peri-implantation rabbit uterus Biology of Reproduction 56 1390-1399

Dunk C, Shams M, Nijjar S, Rhaman M, Qiu Y, Bussolati B and Ahmed A (2000) Angiopoietin-1 and angiopoietin-2 activate trophoblast Tie-2 to promote growth and migration during placental development American Journal of Pathology 156 2185-2199

Dvorak HF (2000) VPF/NEGF and the angiogenic response Seminars in Perinatology 24 75-78

Dvorak HF, Nagy JA, Feng D, Brown LF and Dvorak AM (1999) Vascular permeability factor/vascular endothelial growth factor and the significance of microvascular hyperpermeability in angiogenesis. In Current Topics in Microbiology and Immunology: Vascular Growth Factors and Angiogenesis pp 97-132 Ed. L Claesson-Welsh. SpringerVerlag, Berlin, Heidelberg

Enders A (1993) Overview of the morphology of implantation in primates. In In Vitro Fertilisation and Embryo Transfer in Primates pp 145-157 Eds D Wolf, RL Stouffer and RM Brenner. Springer-Verlag, New York

Fraser HM and Lunn SF (2001) Regulation and manipulation of angiogenesis in the primate corpus luteum Reproduction 121 355-362

Geva E, Ginzinger DG, Zaloudek CJ, Moore DH, Byrne A and Jaffe RB (2002) Human placental vascular development: vasculogenic and angiogenic (branching and nonbranching) transformation is regulated by vascular endothelial growth factor-A, angiopoietin-1, and angiopoietin2 Journal of Clinical Endocrinology and Metabolism 87 4213-4224

Ghosh D, Sharkey AM, Charnock-Jones DS, Dhawan L, Dhara S, Smith SK and Sengupta J (2000) Expression of vascular endothelial growth factor (VEGF) and placental growth factor (PIGF) in conceptus and endometrium during implantation in the Rhesus monkey Molecular Human Reproduction 6 935-941

Goldman-Wohl DS, Ariel I, Greenfield C, Lavy Y and Yagel S (2000) Tie-2 and angiopoietin-2 expression at the fetal-maternal interface: a receptor-ligand model for vascular remodelling Molecular Human Reproduction 6 81-87

Halder JB, Zhao X, Soker S, Paria BC, Klagsbrun M, Das SK and Dey SK (2000) Differential expression of VEGF isoforms and VEGF(164)-specific receptor neuropilin-1 in the mouse uterus suggests a role for VEGF(164) in vascular permeability and angiogenesis during implantation Genesis 26 213-224

Helske S, Vuorela P, Carpen O, Hornig C, Weich $\mathrm{H}$ and Halmesmaki $\mathrm{E}$ (2001) Expression of vascular endothelial growth factor receptors 1, 2 and 3 in placentas from normal and complicated pregnancies Molecular Human Reproduction 7 205-210

Keck PJ, Hauser SD, Krivi G, Sanzo K, Warren T, Feder J and Connolly DT (1989) Vascular permeability factor, an endothelial cell mitogen related to PDGF Science 246 1309-1312

Krussel J, Behr B, Hirchenhain J, Wen Y, Milki AA, Cupisti S, Bielfeld P and Polan ML (2000) Expression of vascular endothelial growth factor mRNA in human preimplantation embryos derived from tripronuclear zygotes Fertility and Sterility $\mathbf{7 4} 1220-1226$

Lobov IB, Brooks PC and Lang RA (2002) Angiopoietin-2 displays VEGFdependent modulation of capillary structure and endothelial cell survival in vivo. Proceedings National Academy of Sciences USA 9911205 11210

Maisonpierre PC, Suri C, Jones PF et al. (1997) Angiopoietin-2, a natural antagonist for Tie2 that disrupts in vivo angiogenesis Science 277 55-60 
Rabbani MM and Rogers PA (2001) Role of vascular endothelial growth factor in endometrial vascular events before implantation in rats Reproduction 122 85-90

Rockwell LC, Pillai S, Olson CE and Koos RD (2002) Inhibition of vascular endothelial growth factor/vascular permeability factor action blocks estrogen-induced uterine edema and implantation in rodents Biology of Reproduction 67 1804-1810

Rowe AJ, Morris KD, Bicknell R and Fraser HM (2002) Angiogenesis in the corpus luteum of early pregnancy in the marmoset and the effects of vascular endothelial growth factor immunoneutralization on establishment of pregnancy Biology of Reproduction 67 1180-1188

Saltzman W, Schultz-Darken NJ, Wegner FH, Wittwer DJ and Abbott DH (1998) Suppression of cortisol levels in subordinate female marmosets: reproductive and social contributions Hormones and Behaviour 33 58-74

Smith SK (2001) Regulation of angiogenesis in the endometrium Trends in Endocrinology and Metabolism 12 147-151

Sugino N, Kashida S, Karube-Harada A, Takiguchi S and Kato H (2002) Expression of vascular endothelial growth factor (VEGF) and its receptors in human endometrium throughout the menstrual cycle and in early pregnancy Reproduction 123 379-387

Suri C, Jones PF, Patan S, Bartunkova S, Maisonpierre PC, Davis S, Sato TN and Yancopoulos GD (1996) Requisite role of angiopoietin-1, a ligand for the TIE2 receptor, during embryonic angiogenesis Cel/ 87 1171-1180

Winther H, Ahmed A and Dantzer V (1999) Immunohistochemical localization of vascular endothelial growth factor (VEGF) and its two specific receptors, Flt- 1 and $\mathrm{KDR}$, in the porcine placenta and nonpregnant uterus Placenta 20 35-43

Wulff C, Wilson H, Largue P, Duncan WC, Armstrong DG and Fraser HM (2000) Angiogenesis in the human corpus luteum: localization and changes in angiopoietins, tie-2, and vascular endothelial growth factor messenger ribonucleic acid Journal of Clinical Endocrinology and Metabolism 85 4302-4309

Wulff C, Wilson H, Rudge JS, Wiegand SJ, Lunn SF and Fraser HM (2001) Luteal angiogenesis: prevention and intervention by treatment with vascular endothelial growth factor trap(a40) Journal of Clinical Endocrinology and Metabolism 86 3377-3386

Wulff C, Wilson H, Dickson SE, Wiegand SJ and Fraser HM (2002) Hemochorial placentation in the primate: expression of vascular endothelial growth factor, angiopoietins, and their receptors throughout pregnancy Biology of Reproduction 66 802-812

Yi XJ, Jiang HY, Lee KK, O WS, Tang PL and Chow PH (1999) Expression of vascular endothelial growth factor (VEGF) and its receptors during embryonic implantation in the golden hamster (Mesocricetus auratus). Cell and Tissue Research 296 339-349

Received 23 January 2003.

First decision 12 March 2003.

Revised manuscript received 4 April 2003.

Accepted 25 April 2003. 